\title{
Playing position specifics of associations between running performance during the training and match in male soccer players
}

\author{
Toni Modric ${ }^{1,2}$, Sime Versic ${ }^{2}$, and Damir Sekulic ${ }^{2, *}$ \\ ${ }^{1} H N K$ Hajduk Split, Split, Croatia; and ${ }^{2}$ Faculty of Kinesiology, University of Split, Split, Croatia
}

Copyright: (C) 2020 T. Modric et al. This is an open access article licensed under the Creative Commons Attribution License (https://creativecommons.org/licenses/by/4.0/).

\begin{abstract}
Background: The relationship between the training load and game load considering soccer playing positions has not been sufficiently examined. Objective: The aim of this study was to examine the position-specific associations between running performance (RP) during the training and match in professional-level male soccer. Methods: The RPs of 15 players (age $23.57 \pm 2.84$ years, body height $181.9 \pm 5.17 \mathrm{~cm}$, body mass $78.36 \pm 4.18 \mathrm{~kg}$ ) were measured by the Global Positioning System over one half-season of the highest-level Croatian soccer competition and assessed according to their playing positions: central defenders $(n=22)$, fullbacks $(n=23)$, wide midfielders $(n=29)$, wingers $(n=6)$, and forwards $(n=12)$. Variables included the total distance covered; the distance covered by low intensity running, running, high-speed running, sprinting, and high intensity running; and the number of accelerations, high-intensity accelerations (HIA), decelerations, and high-intensity decelerations (HID). Results: Analysis of variance revealed significant differences in high-speed running, HID, and HIA ( $p=.01$, all highest in forwards), and sprinting ( $p=.01$, highest in the wide midfielders). The HIA and HID at training were significantly ( $p=.04$ and .01 , respectively) correlated ( $r=.42$ and .52$)$ with the corresponding match RP in fullbacks. The high-intensity running (high-speed running + sprinting), running, HIA and HID performance values were significantly correlated $(r=.64, .52, .59$, and .52 , respectively; all $p=.01$ ) with the corresponding running performances from matches in central midfielders. Conclusions: Structure-specific training is highly recommended for some playing positions. The information obtained can be used to improve soccer training programs.
\end{abstract}

Keywords: football, training load, game load, relationships, conditioning, performance analysis

\section{Introduction}

Soccer is a highly complex team sport; it involves two teams, changing dynamics and multistructural movements (Modric, Versic, Sekulic, \& Liposek, 2019). The actions during a game involve running at different intensity levels, many changes in direction, tackles and jumps with short recovery periods (Sládečková, Botek, Krejčí, \& Lehnert, 2019). Professional soccer training programs are designed to enable players to achieve an appropriate conditioning status, prevent injuries and compete at the highest possible level during the season (Jaspers, Brink, Probst, Frencken, \& Helsen, 2017). For such purposes, training programs need to be able to be modified so that they are optimally individualized and that, consequently, players' match performance is

\footnotetext{
* Address for correspondence: Damir Sekulic, Faculty of Kinesiology, University of Split, Teslina 6, 21000 Split, Croatia. E-mail: dado@kifst.hr
}

maximized (Castagna, Chamari, Stolen, \& Wisloff, 2005). Thus, proper and continuous monitoring of the training load is important to determine the applied training load and implement interventions in subsequent sessions (i.e., increases or decreases in the training load; Jaspers et al., 2017; Rebelo et al., 2012).

Training loads can comprise external and internal loads (Impellizzeri, Rampinini, Coutts, Sassi, \& Marcora, 2004). External training loads are measured by the work done by players and are currently commonly monitored by Global Positioning System (GPS) technology (Buchheit et al., 2013). Internal training loads are measured by the metabolic demands required to complete the external work (Bingham, 2015) and are commonly monitored using physiological and/or perceptual measures such as the player's heart rate and rating of perceived exertion (Coutts \& Cormack, 2014). However, the external load (i.e., total distance covered, distances covered in different speed zones, number of accelerations and decelerations) is most 
often quantified for a comprehensive understanding of the soccer training program implemented, and there is a growing interest in the monitoring of the external training loads during soccer training (Akenhead \& Nassis, 2016; Carling \& Orhant, 2010; Jaspers et al., 2017; Owen et al., 2015; Stevens, de Ruiter, Twisk, Savelsbergh, \& Beek, 2017).

In a very recent study, the authors assessed the in-season external training load of the UEFA Champions League team and indicated that the total distance tended to decrease during the in-season (from $5,589 \mathrm{~m}$ to $4,545 \mathrm{~m}$; Oliveira et al., 2019). These results are consistent with those in reports on training programs for elite English Premier League soccer players (Malone et al., 2015). Akenhead, Harley, and Tweddle (2016) assessed the position-specific external training loads of an English Premier League soccer team during training, especially in terms of acceleration, and found that some acceleration variables successfully differentiated playing positions, with midfield players accounting for a large portion of the total distance covered and central defenders being differentiated by low and moderate acceleration thresholds. Anderson et al. (2016) quantified training loads in English Premier League soccer players during a one-, two- and three-game week schedule and indicated that the total distance covered in daily training sessions was lower in the three-game week than in the one- and two-game weeks.

The match performance in official soccer matches (i.e., external match loads) was more frequently evaluated than were training loads; additionally, the results obtained by highly sophisticated global positioning systems (Aquino et al., 2020; Modric et al., 2019) and those obtained by various video-based platforms are generally consistent (Barros et al., 2007; Bradley et al., 2009; Di Salvo et al., 2007; Liu, Yi, Giménez, Gómez, \& Lago-Peñas, 2015; Vigne, Gaudino, Rogowski, Alloatti, \& Hautier, 2010). Accordingly, it is evident that both (i) external training load and (ii) match performance during soccer matches are often investigated separately (Jaspers et al., 2017; Sarmento et al., 2014), while the relationship between the external match load in official matches and weekly external training load has not been sufficiently explored. To the best of our knowledge, only two studies have examined players' match performance in official matches in relation to the in-season training periodization of elite soccer teams.

Stevens et al. (2017) compared the in-season external training load and match load in professional Dutch Eredivisie soccer players and reported that there was a lower load when training approached the match day Additionally, regarding the relative match values, the weekly acceleration training load was higher (3.1-3.9 times) than the running zone training load and high-speed running training load (2.5 and 2.1 times, respectively). In a very recent study, Clemente et al. (2019) investigated the general relationship between total weekly training loads and the weekly match demands in the Primeira Liga and reported trivial-tosmall correlations between the total weekly loads and match demands.

Although some previous studies evidenced the correlations between external match load and training load (Clemente et al., 2019; Stevens et al., 2017), to the best of our knowledge, taking into account soccer playing positions, there is no information about the relationship between the training load and game load. We were of the opinion that this topic is important to study since each playing position is associated with specific duties during a game (Kim, 2000), and these specific duties are naturally influenced by positionspecific indicators of the game load (Dellal, Wong, Moalla, \& Chamari, 2010). Therefore, the main aim of this study was to examine the position-specific associations that may exist between the external training load in one week and match performance in subsequent soccer matches. Additionally, the differences in running performance among (i) playing positions for the total training load in the observed period (e.g., half-season - 15 weeks) and (ii) in each mesocycle (the first three mesocycles lasted 4 weeks, while the last one lasted 3 weeks) were analyzed as suggested previously (Oliveira et al., 2019).

\section{Methods}

\section{Participants and design}

For the purpose of this research, all participants signed an informed consent form agreeing to participate in the study. Fifteen players were analyzed through 92 match performances and running performance events that occurred during training sessions in the week preceding each match. Averagely, 6 match and running performances were analyzed per each player (ranged from 1 to 12). All the data were collected from the 15 matches of Croatian top-division competitive soccer and from 75 training sessions during one half-season in which matches were played. The half season phase was analyzed through 4 mesocycles, as suggested previously by Oliveira et al. (2019). First three mesocycles lasted 4 weeks, while the last one lasted 3 weeks. The players $(M \pm S D$, age $23.57 \pm 2.84$ years, body height $181.9 \pm 5.17 \mathrm{~cm}$, body mass $78.36 \pm 4.18 \mathrm{~kg}$ ) were members of one team. In this study players' performances were observed according to their playing positions, and resulted in performances at the position of (i) central defender (CD, $n=22$ ), (ii) fullbacks 
(FB, $n=23$ ), (iii) central midfielders (CM, $n=29)$, (iv) winger-midfielders (WM, $n=6$ ), and forwards (FW, $n=12$ ).

At the end of the observed period, the team was ranked 2nd of 10 teams. Only the performance of the players who played the whole game and participated in all training sessions in the week before each match were included in this study.

The study protocol was approved by the Ethical Board of University of Split, Faculty of Kinesiology.

\section{Procedures}

The variables in this study included players' age, body height, weight and playing position and two sets of running performance variables, namely, running performance variables for the official matches (RMs) and running performance variables measured during training in the preceding week (RTs). Specifically, the RMs were measured during official matches, as previously suggested and reported in detail (Oliveira et al., 2019). The RTs were measured during all the training sessions that the team participated in during the week and preceded official matches. The RTs and RMs included the total distance covered during the match/training week $(\mathrm{m})$; distance covered in five speed categories: low intensity running $(<14.3$ $\mathrm{km} / \mathrm{h}$ ), running (14.4-19.7 km/h), high-speed running (19.8-25.1 km/h), sprinting $(\geq 25.2 \mathrm{~km} / \mathrm{h})$, and high-intensity running $(>19.8 \mathrm{~km} / \mathrm{h})$; total accelerations $\left(>0.5 \mathrm{~m} / \mathrm{s}^{2}\right)$; high-intensity accelerations $(>3$ $\left.\mathrm{m} / \mathrm{s}^{2}\right)$; total decelerations $\left(<[-] 0.5 \mathrm{~m} / \mathrm{s}^{2}\right)$; and highintensity decelerations $\left(<[-] 3 \mathrm{~m} / \mathrm{s}^{2}\right)$.

All data were collected by GPS technology (OptimEye S5 \& X4, Catapult, Melbourne, Australia) with a sampling frequency of $10 \mathrm{~Hz}$. The average number of satellite signals was $12.03 \pm 0.5$, while the horizontal dilution of precision was $0.83 \pm 0.1$. The reliability and validity of the equipment has previously been presented in detail (Castellano, Casamichana, Calleja-González, San Román, \& Ostojic, 2011; Johnston, Watsford, Kelly, Pine, \& Spurrs, 2014).

\section{Statistics}

The normality of the distributions was confirmed by the Kolmogorov-Smirnov test, and the data are presented as the means \pm standard deviations. Homogeneity was checked by Levene's test. Differences among the playing positions in terms of running performance were analyzed by one-way analysis of variance, while the consecutive Scheffe post hoc test was used to analyze the specific differences across playing positions. To evaluate the effect sizes (ES), partial eta-squared values were presented $(>.02$, small; $>.13$, medium; $>.26$, large; Cohen, 1988; Ferguson, 2009).
To identify the associations in the running parameters between training sessions and games, Pearson's correlation coefficient was calculated for the observed half-season period.

For all analyses, Statistica (Version 13; TIBCO Software, Palo Alto, CA, USA) was used, and a significance level $\alpha=.05$ was applied.

\section{Results}

Table 1 presents the descriptive parameters for the running performance in weekly training sessions and the corresponding differences among playing positions. The highest amount of high-intensity running (highspeed running + sprinting) was performed by FBs (732 $\mathrm{m}$; significant post hoc difference between FBs and CMs; all $p<.01$ ), with medium ES. The number of high-intensity decelerations was significantly lower in the CMs (37 repetitions) than in the CDs (63 repetitions) and FWs (71 repetitions), all $p<.01$, large ES. The number of high-intensity accelerations was significantly higher in the FWs, CDs and FBs (37, 32 and 26 repetitions, respectively) than in the CMs (16 repetitions), $p<.05$, large ES.

Table 2 presents descriptive parameters for the match performance and the corresponding differences among playing positions. The greatest total distance was covered by CMs $(10,951 \mathrm{~m}$; significantly higher than that of FBs, CDs and FWs; all $p<.01$, large ES). Compared to all other playing positions, CDs had the lowest high-intensity distance covered $(432 \mathrm{~m}$, large ES) and distance covered in the zone of running (958 $\mathrm{m}$, large ES). CMs carried out the highest number of total accelerations (478 repetitions, large ES) and decelerations (473 repetitions, large ES), which was both significantly higher than that of FBs, CDs and FWs (all $p<.01)$. The number of high-intensity accelerations (31 repetitions, large ES) and decelerations (52 repetitions, large ES) was significantly higher for FWs than for all other playing positions (all $p<.01$ ).

Figure 1 presents training loads across playing positions in four mesocycles. In the first mesocycle (Figure 1A), significant differences among the playing positions were found for high-intensity running (sprinting + high-speed running) and the number of high-intensity accelerations/decelerations (all were highest in the FWs). In the second mesocycle (Figure 1B), significant differences were found in high-intensity accelerations and decelerations (which were the highest in the CDs and FWs). In the fourth mesocycle (Figure 1D), the playing positions differed by the sprint distance covered (which was the highest in the WMs and FWs). 

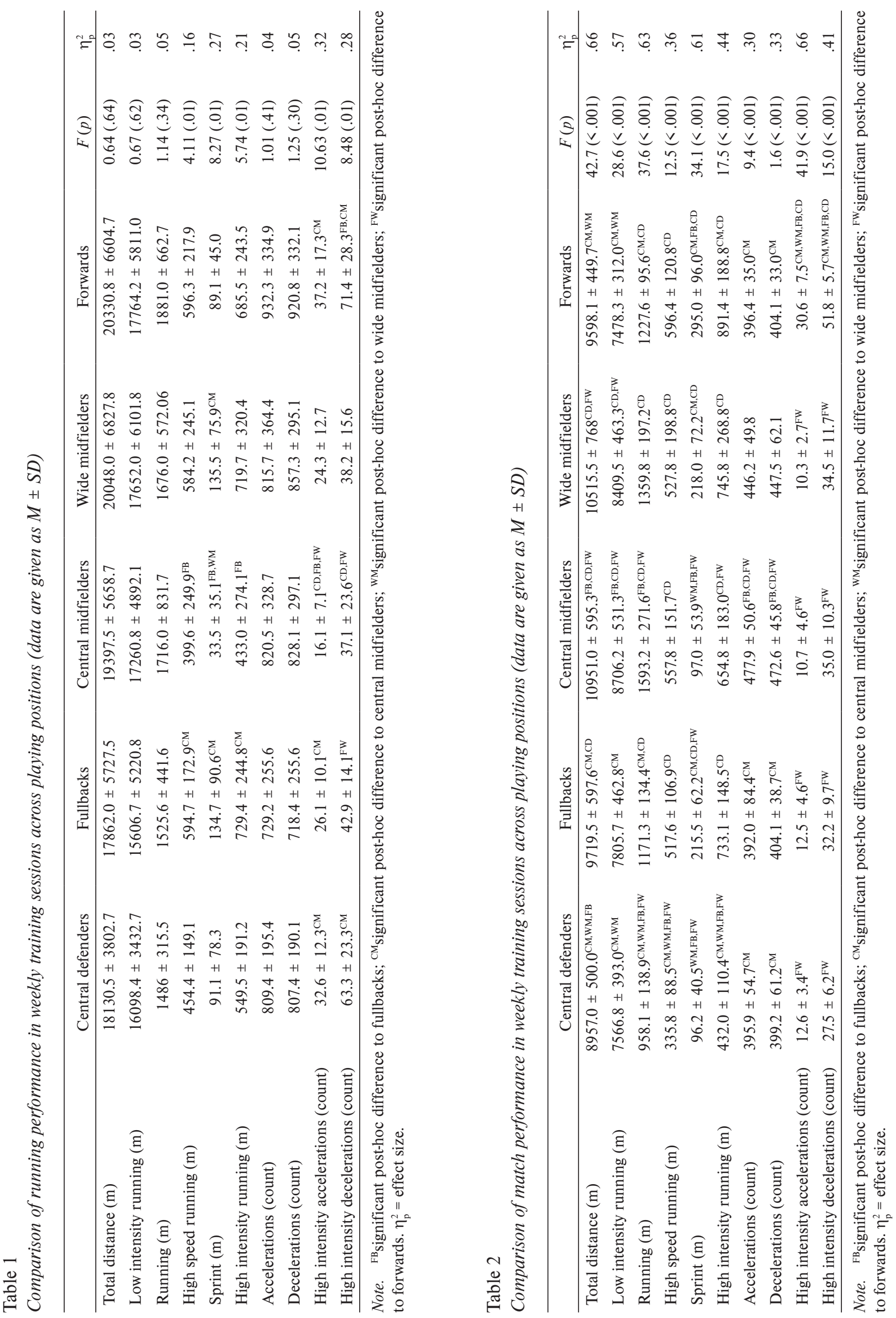
A

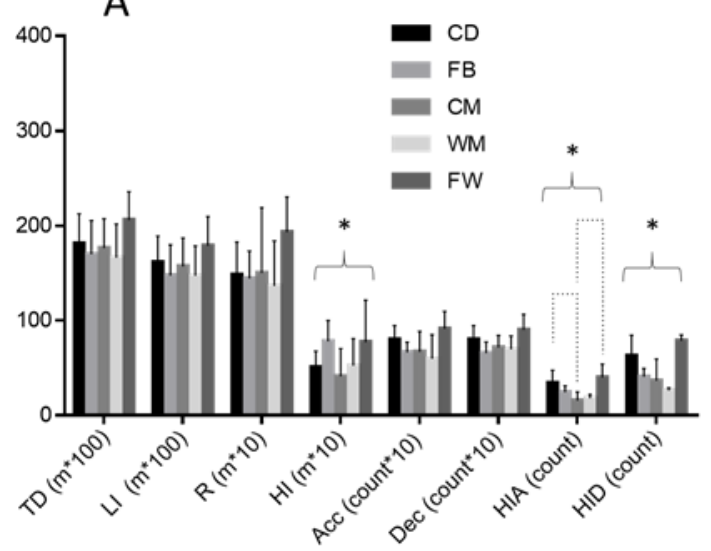

C

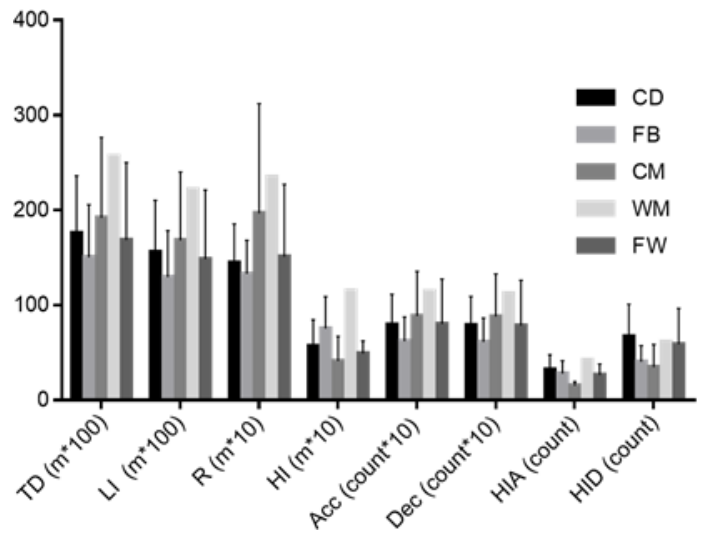

B

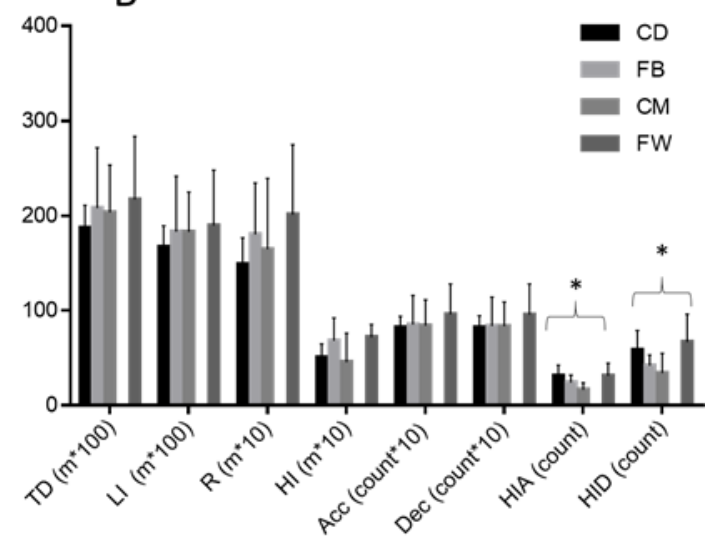

D

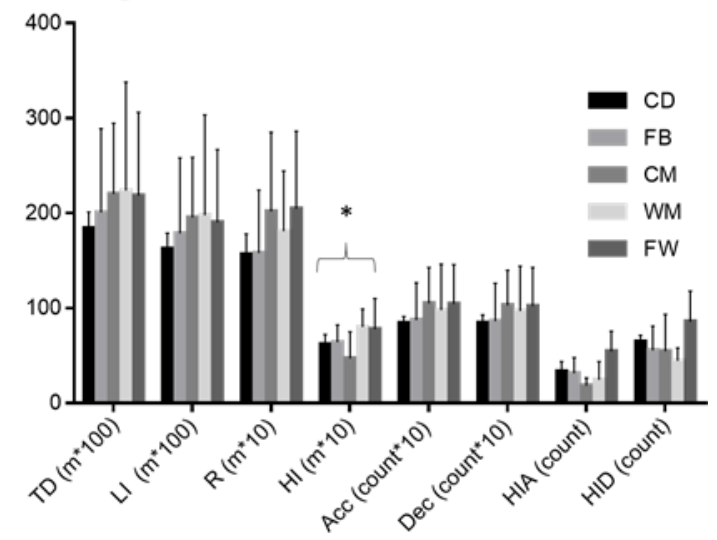

Figure 1. Differences among the playing positions $(\mathrm{CD}=$ central defenders, $\mathrm{FB}=$ fullbacks, $\mathrm{CM}=$ central midfielders, $\mathrm{WM}=$ wide midfielders, $\mathrm{FW}=$ forwards $)$ in the running performance during training in each mesocycle $(\mathrm{A}=$ first mesocycle, $\mathrm{B}=$ second mesocycle, $\mathrm{C}=$ third mesocycle, $\mathrm{D}=$ fourth mesocycle). $\mathrm{TD}=$ total distance covered, $\mathrm{LI}=$ low intensity running, $\mathrm{R}=$ running, $\mathrm{HI}=$ high intensity running (high speed + sprint $),$ Acc $=$ accelerations, Dec $=$ decelerations, $\mathrm{HIA}=$ high intensity accelerations, HID = high intensity decelerations. *denotes significant differences among playing positions obtained by analysis of variance. Dashed lines present significant post-hoc differences.

Table 3 presents the correlations between match performance and weekly values of the same variables (e.g., corresponding variables/performance). The FBs' high-intensity accelerations and decelerations in matches were significantly correlated ( $p=.04$ and .01 , respectively) with corresponding variables obtained in the previous week during training ( $r=.42$ and .52 for accelerations and decelerations, respectively). For the CDs, the distance covered in the zone of running and high-intensity accelerations in the matches were found to be correlated with the corresponding training variables ( $r=.51$ and .48 , respectively). The CMs' distance covered during high-intensity running (high speed running + sprinting; $r=.64)$, distance covered during running $(r=.52)$, and number of high-intensity accelerations/ decelerations ( $r=.59$ and .63 , respectively) were found to be significantly correlated with the corresponding performance in the training sessions (all $p=.01$ ).

\section{Discussion}

\section{Differences among playing positions in running performance}

The present study demonstrated that the FWs tended to cover the longest total distance $(20,330 \mathrm{~m}$ on average per week), and the FBs covered the shortest total distance $(17,862 \mathrm{~m}$ on average), but there was no significant difference across the playing positions in terms of the weekly total distance covered. These findings are in line with those in the previously cited Portuguese study in which the authors demonstrated limited relevant variation among the playing positions (Oliveira et al., 2019). This result may suggest that immediately after the preseason, coaches still emphasize physical conditioning for certain players and consequently implement position-specific training loads only at the very beginning of the competitive season. 
Table 3

Pearson's correlation coefficients between match performance and weekly training performance of the corresponding variables for the observed half-season (data are given as $r(p)$ )

\begin{tabular}{lccccc}
\hline & $\begin{array}{c}\text { Central defenders } \\
(n=22)\end{array}$ & $\begin{array}{c}\text { Fullbacks } \\
(n=23)\end{array}$ & $\begin{array}{c}\text { Central midfield- } \\
\text { ers }(n=29)\end{array}$ & $\begin{array}{c}\text { Wide midfielders } \\
(n=6)\end{array}$ & $\begin{array}{c}\text { Forwards } \\
(n=12)\end{array}$ \\
\hline Total distance & $.01(.95)$ & $.06(.78)$ & $.34(.06)$ &. $.14(.78)$ & $.10(.74)$ \\
Low intensity running & $-.13(.55)$ & $-.08(.72)$ & $.20(.28)$ & $-.10(.84)$ & $.25(.42)$ \\
Running & $.51(.02)$ & $.08(.72)$ & $.52(.01)$ & $.17(.74)$ & $.02(.94)$ \\
High speed running & $.47(.03)$ & $.20(.35)$ & $.56(.01)$ & $.41(.42)$ & $-.25(.44)$ \\
Sprint & $.16(.49)$ & $.01(.99)$ & $.64(.01)$ & $.30(.56)$ & $.17(.59)$ \\
High intensity running & $.54(.01)$ & $.17(.41)$ & $.64(.01)$ & $.37(.46)$ & $-.21(.50)$ \\
Total accelerations & $.14(.52)$ & $.01(.99)$ & $.23(.21)$ & $.28(.58)$ & $.23(.47)$ \\
Total decelerations & $.20(.36)$ & $.23(.28)$ & $.17(.35)$ & $.44(.38)$ & $.33(.29)$ \\
High intensity accelerations & $.48(.02)$ & $.42(.04)$ & $.59(.01)$ & $.08(.87)$ & $.42(.17)$ \\
High intensity decelerations & $.33(.13)$ & $.52(.01)$ & $.63(.01)$ & $.47(.35)$ & $.29(.35)$ \\
\hline
\end{tabular}

The external players (FBs and WMs) and front players (FWs) observed herein covered the greatest distance during high-intensity running in training sessions $(\mathrm{FB}=729 \mathrm{~m}, \mathrm{WM}=719 \mathrm{~m}, \mathrm{FW}=685 \mathrm{~m}$, followed by $\mathrm{CD}=545 \mathrm{~m}$ and $\mathrm{CM}=433 \mathrm{~m}$ ), which is logical considering that similar patterns are observed in soccer games (Bradley et al., 2009; Di Salvo et al., 2007; Mallo, Mena, Nevado, \& Paredes, 2015). Additionally, we found significant differences between FBs and $\mathrm{CMs}$ in the high-intensity running distance covered during training, which indicates that different coaching approaches and position-specific training were implemented. This result supports the recent considerations of different physical requirements and training demands for each playing position (Oliveira et al., 2019). For instance, the main tactical requirements for FBs include the number of entries to the third part of the pitch (i.e., presses) and the number of crosses (van Lingen, 1997; Yi, Jia, Liu, \& Gómez, 2018). Therefore, specific training for this playing position should involve more high-intensity running than $\mathrm{CM}$ training, as the main role of CMs is to organize the offense by facilitating ball control and passes rather than by invading the opponent's area (Yi et al., 2018).

Analysis of the weekly total number of accelerations and decelerations in training did not indicate differences across playing positions. On the other hand, the CMs' weekly number of high-intensity accelerations in training was found to be statistically significantly lower than those for nearly all other playing positions. Additionally, the number of high-intensity decelerations in training was found to be statistically significantly lower in CMs than in CDs and FWs. These findings, together with the previously reported results of the high-intensity distance covered each week, imply that CMs train at a relatively low intensity throughout the week. These players are probably more focused on ball possession and the number of key passes, dribbles and shots, considering that these performance variables are key for their success in soccer games (Modric et al., 2019).

When the total training load was assessed through mesocycles, there were significant differences across playing positions for training running performance in almost all mesocycles. These results are not in accordance with those in the very recent study performed by Oliveira et al. (2019), in which the authors demonstrated that there are significant differences among playing positions only in the first mesocycle after the preseason. Presumably, this inconsistency may be a result of the different training approaches and philosophies implemented in different countries (e.g., Portugal and Croatia). Regardless of the differences in the results between studies, it is clear that in the mesocycles where significant differences among positions were found, the training sessions involved positionspecific training drills. In contrast, through the weeks in which there were no significant differences, the coaches actually used a similar training methodology for all players, independent of their playing positions in the matches.

\section{Correlations between training load and game performance}

Our results indicated that the distance covered while running (speed of 14.4-19.7 km/h) and number of high-intensity accelerations in matches are correlated with the weekly values of the same variables for CDs. In other words, training demands that involve (i) more effort in the running zone and (ii) high-intensity 
accelerations correspond to changes in the same performance variables during games for this playing position. As previously mentioned, these variables were identified as important determinants of game success for this position (Modric et al., 2019). Therefore, the correlation between training sessions and matches in these variables implies that (i) structurally position-specific training has been applied in training programs for CDs and that (ii) such an approach directly corresponded to their performance during games. As a result, along with specific soccer requirements, it is highly recommended that position-specific training for CDs involve training in the running zone and high-intensity accelerations, which can consequently improve the success of CDs in soccer games.

Of all the positions, central midfielders covered the shortest distance during high-intensity running (highspeed running [19.8-25.1 km/h] + sprinting [ $\geq 25.2$ $\mathrm{km} / \mathrm{h}]$ ) and had the fewest high-intensity accelerations and decelerations during training. Accordingly, this result may suggest that CMs are more focused on developing soccer skills than performing high-intensity tasks. This result is consistent with those in previous studies on match performance (Modric et al., 2019). Additionally, it must be noted that the Croatian First Football (Soccer) League is less competitive than the top-level European leagues (Modric et al., 2019), so it seems that high-intensity efforts in CM training and the style of the games are probably sufficient for position-specific success in the competitive soccer league observed in this study (Croatian National League). However, the presence of a positive correlation between high-intensity efforts (i.e., correlation between distance covered during high-intensity running and number of high-intensity accelerations and decelerations) achieved during training sessions and games indicates that training focused on high-intensity efforts may lead to improvement in CMs' high-intensity match performance in the same week. This relation is important for creating position-specific training programs for the central midfielders that are planning to play in top-level European soccer leagues.

The sprint distance covered during games is an important determinant of FWs' overall game performance (Modric et al., 2019). In other words, the greater the distance covered while sprinting, the higher the position-specific performance of these players will be during games. Our results indicated that FWs cover the greatest distance during high-intensity running (i.e., both high speed and sprinting distances) during the weekly training sessions (please see previous discussion for details), but surprisingly, no correlations between any of the external load variables observed in training and during matches for this position were observed. Although correlations for FWs possibly did not reach statistical significance because of the relatively small number of running performances $(n=12)$, this result is particularly interesting and important to highlight, as sprinting is an important determinant of their success. Because the authors of this study have been deeply involved in the training and conditioning of the players included in this study, we suggest that the reason for this finding is specific (i.e., a lack of correlation between sprinting in training sessions and sprinting in games). In brief, the main objective of FWs is to score goals, which is also the main objective in soccer matches (Kim, 2000). To improve their scoring skills, FW players are usually more involved than other players in position-specific work, which mostly consists of different finishing drills (i.e., shooting, scoring goals). In fact, at the end of most team training sessions, FWs stay for extra practice. However, their training drills are based on short-intensity efforts (high-intensity accelerations and decelerations + shooting) rather than sprinting + shooting. The differences among playing positions directly support this explanation (FWs had the most high-intensity accelerations/ decelerations of all playing positions; see Results for details). Accordingly, it seems that sprinting, although it is an important determinant of FW success, is actually undertrained in weekly training sessions.

Previously, it was discussed that performing more stops (i.e., decelerations) negatively affects FBs' success in soccer games (Modric et al., 2019). Specifically, it was suggested that more decelerations alter FBs' ability to perform attacks, crosses and moves to the third part of the pitch (i.e., presses), which are all important determinants of successful performance for players in this position (Modric et al., 2019). In general, soccer players with higher maximum acceleration rates can jump higher, sprint faster (over short distances), and achieve changes in direction at higher velocities (Loturco et al., 2019). Thus, to achieve more presses (i.e., number of entries to the third part of the pitch), higher maximum acceleration and deceleration rates may enable FBs to have an advantage over the opponent defenders and make more crosses. Taking this concept into account, the positive correlation between the number of high-intensity accelerations and decelerations during the matches and weekly training sessions may suggest that the training for FBs was specifically structured. Evidently, performing more high-intensity accelerations and decelerations in a week will positively affect the number of high-intensity accelerations and decelerations that FBs achieve during matches. Such training may decrease the total number of decelerations performed in matches and consequently improve the 
performance of FBs in particular; however, this topic should be explored in more detail in future studies.

The present study did not show a correlation between running performance variables observed in matches and weekly training sessions for WMs. Further, a small sample for this position $(n=6)$ contributed to statistical significance not being reached. However, this result is not surprising considering that previous position-specific analyses have indicated that there is no correlation between WMs' match performance and general performance in matches (Modric et al., 2019). Collectively, these results suggest that the performance of WMs is based on soccer skills, both during training and matches. Specifically, although WMs generally experience the highest physical requirements during matches and training (Mallo et al., 2015; Oliveira et al., 2019), it seems that it is more important for them to be focused on soccerrelated skills (i.e., passing, dribbling, shooting) rather than running performance; running performance is actually a necessary prerequisite (i.e., basis) and not a factor that directly influences their performance.

\section{Limitations and strengths of the study}

This study has several limitations that must be mentioned before drawing the conclusion. First, the study evaluated a team competing in the Croatian National Championship, which is certainly not the top-level European competition. Therefore, the results are generalizable to similar competitive levels. Next, we observed only one half-season, and for more extensive analysis, evaluation of the whole competitive season is necessary. Additionally, in this study, we included only players who participated in all training sessions and played the whole game in each week, which reduced the number of observations. However, this approach was done intentionally since otherwise, the running performance results would be logically influenced by numerous other factors apart from those related to training load and game performance. Last, due to the limited number of entities, the associations were not analyzed for different mesocycles (i.e., we observed only those players who participated in all training sessions and played the whole game during each week over the observed half-season).

This is probably the first study where the running performance at training and during games was studied separately for playing positions. Therefore, we believe that the findings can be used to improve the training process and consequently increase the possibility of achieving positive competitive results in soccer. Additionally, the present study is the first to utilize position-specific external training load measures to assess changes in the match performance of elite soccer players while at the same time reinforcing the importance of a load monitoring system in elite sporting environments. Ultimately, the results of this study can be used to change the current monitoring approaches and lead to a better understanding of position-specific demands.

\section{Conclusions}

This study suggests that training sessions based on high-intensity accelerations and efforts during running likely positively affect related performance variables measured during $\mathrm{m}$ atches for $\mathrm{CDs}$, which consequently increase their success in soccer. Additionally, FW players should perform more sprinting-related training during the week, as it has previously been indicated to be an important determinant of their success in soccer.

Training for CMs and WMs should be based more on soccer skills rather than running performance. However, although CMs' success in soccer is not dependent on their match performance, it should be emphasized that increasing the training intensity can probably positively affect their performance in matches.

The results suggest that structure-specific training is highly recommended for CD and FW players, while the relationship between weekly external loads and running match performance in FB players should be explored in further detail in future studies. Furthermore, the results of this study can be used to improve the training process and consequently increase the performance of the whole team.

Finally, this study did not show significant differences between playing positions during the weekly training sessions in variables that determine training volume (e.g., the total distance covered, distance covered during low-intensity and moderate-intensity running, total number of accelerations/decelerations), while the intensity variables (e.g., distance covered during high-speed running and sprinting, number of highintensity accelerations/decelerations) could be used to differentiate the players by playing positions.

\section{Conflict of interest}

There were no conflicts of interest.

\section{References}

Akenhead, R., Harley, J. A., \& Tweddle, S. P. (2016). Examining the external training load of an English Premier 
League football team with special reference to acceleration. Journal of Strength and Conditioning Research, 30, 2424-2432.

Akenhead, R., \& Nassis, G. P. (2016). Training load and player monitoring in high-level football: Current practice and perceptions. International Journal of Sports Physiology and Performance, 11, 587-593.

Anderson, L., Orme, P., Di Michele, R., Close, G. L., Morgans, R., Drust, B., \& Morton, J. P. (2016). Quantification of training load during one-, two-and three-game week schedules in professional soccer players from the English Premier League: Implications for carbohydrate periodisation. Journal of Sports Sciences, 34, 1250-1259.

Aquino, R., Carling, C., Palucci Vieira, L., Martins, G., Jabor, G., Machado, J., \& Puggina, E. (2020). Influence of situational variables, team formation, and playing position on match running performance and social network analysis in Brazilian professional soccer players. Journal of Strength and Conditioning Research, 34, 808-817.

Barros, R. M., Misuta, M. S., Menezes, R. P., Figueroa, P. J., Moura, F. A., Cunha, S. A., ... Leite, N. J. (2007). Analysis of the distances covered by First Division Brazilian soccer players obtained with an automatic tracking method. Journal of Sports Science and Medicine, 6, 233-242.

Bingham, G. (2015). The impact of training loads on in-match soccer performance variables: A position-based case report (Unpublished master thesis). East Tennessee State University, Johnson City, TN.

Bradley, P. S., Sheldon, W., Wooster, B., Olsen, P., Boanas, P., \& Krustrup, P. (2009). High-intensity running in English FA Premier League soccer matches. Journal of Sports Sciences, 27, 159-168.

Buchheit, M., Racinais, S., Bilsborough, J., Bourdon, P., Voss, S., Hocking, J., ... Coutts, A. (2013). Monitoring fitness, fatigue and running performance during a pre-season training camp in elite football players. Journal of Science and Medicine in Sport, 16, 550-555.

Carling, C., \& Orhant, E. (2010). Variation in body composition in professional soccer players: Interseasonal and intraseasonal changes and the effects of exposure time and player position. Journal of Strength and Conditioning Research, 24, 1332-1339.

Castagna, C., Chamari, K., Stolen, T., \& Wisloff, U. (2005). Physiology of soccer: An update. Sports Medicine, 35, 501-536.

Castellano, J., Casamichana, D., Calleja-González, J., San Román, J., \& Ostojic, S. M. (2011). Reliability and accuracy of $10 \mathrm{~Hz}$ GPS devices for short-distance exercise. Journal of Sports Science and Medicine, 10, 233-234.

Clemente, F. M., Rabbani, A., Conte, D., Castillo, D., Afonso, J., Clark, T., ... Knechtle, B. (2019). Training/ match external load ratios in professional soccer players: A full-season study. International Journal of Environmental Research and Public Health, 16, 3057.

Cohen, J. (1988). Statistical power analysis for the behavioral sciences (2nd ed). New York, NY: Lawrence Erlbaum Associates.

Coutts, A. J., \& Cormack, S. (2014). Monitoring the training response. In D. Joyce \& D. Lewindon (Eds.), Highperformance training for sports (pp. 71-84). Champaign, IL: Human Kinetics.
Dellal, A., Wong, D. P., Moalla, W., \& Chamari, K. (2010). Physical and technical activity of soccer players in the French First League - with special reference to their playing position. International SportMed Journal, 11, 278-290.

Di Salvo, V., Baron, R., Tschan, H., Montero, F. C., Bachl, N., \& Pigozzi, F. (2007). Performance characteristics according to playing position in elite soccer. International SportMed Journal, 28, 222-227.

Ferguson, C. J. (2009). An effect size primer: A guide for clinicians and researchers. Professional Psychology: Research and Practice, 40, 532-538.

Impellizzeri, F. M., Rampinini, E., Coutts, A. J., Sassi, A., \& Marcora, S. M. (2004). Use of RPE-based training load in soccer. Medicine \& Science in Sports \& Exercise, 36, 1042-1047.

Jaspers, A., Brink, M. S., Probst, S. G., Frencken, W. G., \& Helsen, W. F. (2017). Relationships between training load indicators and training outcomes in professional soccer. Sports Medicine, 47, 533-544.

Johnston, R. J., Watsford, M. L., Kelly, S. J., Pine, M. J., \& Spurrs, R. W. (2014). Validity and interunit reliability of $10 \mathrm{~Hz}$ and $15 \mathrm{~Hz}$ GPS units for assessing athlete movement demands. Journal of Strength and Conditioning Research, 28, 1649-1655.

Kim, Y. (2000). A fitness profiles of the professional soccer players by each position. Korean Journal of Sports Medicine, 18, 217-226.

Liu, H., Yi, Q., Giménez, J.-V., Gómez, M.-A., \& LagoPeñas, C. (2015). Performance profiles of football teams in the UEFA Champions League considering situational efficiency. International Journal of Performance Analysis in Sport, 15, 371-390.

Loturco, I., Pereira, L. A., Freitas, T. T., Alcaraz, P. E., Zanetti, V., Bishop, C., \& Jeffreys, I. (2019). Maximum acceleration performance of professional soccer players in linear sprints: Is there a direct connection with change-ofdirection ability? PLOS ONE, 14, e0216806.

Mallo, J., Mena, E., Nevado, F., \& Paredes, V. (2015). Physical demands of top-class soccer friendly matches in relation to a playing position using global positioning system technology. Journal of Human Kinetics, 47, 179-188.

Malone, J. J., Di Michele, R., Morgans, R., Burgess, D., Morton, J. P., \& Drust, B. (2015). Seasonal training-load quantification in elite English Premier League soccer players. International Journal of Sports Physiology and Performance, 10, 489-497.

Modric, T., Versic, S., Sekulic, D., \& Liposek, S. (2019). Analysis of the association between running performance and game performance indicators in professional soccer players. International Journal of Environmental Research and Public Health, 16, 4032.

Oliveira, R., Brito, J. P., Martins, A., Mendes, B., Marinho, D. A., Ferraz, R., \& Marques, M. C. (2019). In-season internal and external training load quantification of an elite European soccer team. PLOS ONE, 14, e0209393.

Owen, A. L., Forsyth, J. J., Wong, D. P., Dellal, A., Connelly, S. P., \& Chamari, K. (2015). Heart rate-based training intensity and its impact on injury incidence among elitelevel professional soccer players. Journal of Strength and Conditioning Research, 29, 1705-1712. 
Rebelo, A., Brito, J., Seabra, A., Oliveira, J., Drust, B., \& Krustrup, P. (2012). A new tool to measure training load in soccer training and match play. International Journal of Sports Medicine, 33, 297-304.

Sarmento, H., Marcelino, R., Anguera, M. T., Campaniço, J., Matos, N., \& Leitão, J. C. (2014). Match analysis in football: A systematic review. Journal of Sports Sciences, 32, 1831-1843.

Sládečková, B., Botek, M., Krejčí, J., \& Lehnert, M. (2019). Assessment of the body response to specific fatigue exercise protocol SAFT90 in U16 soccer players. Acta Gymnica, 49, 157-163.

Stevens, T. G., de Ruiter, C. J., Twisk, J. W., Savelsbergh, G. J., \& Beek, P. J. (2017). Quantification of in-season training load relative to match load in professional Dutch Eredivisie football players. Science and Medicine in Football, 1, 117-125.

van Lingen, B. (1997). Coaching soccer: The official coaching book of the Dutch Soccer Association. Spring City, PA: Reedswain.

Vigne, G., Gaudino, C., Rogowski, I., Alloatti, G., \& Hautier, C. (2010). Activity profile in elite Italian soccer team. International Journal of Sports Medicine, 31, 304-310.

Yi, Q., Jia, H., Liu, H., \& Gómez, M. Á. (2018). Technical demands of different playing positions in the UEFA Champions League. International Journal of Performance Analysis in Sport, 18, 926-937. 